\title{
COVID-19 Stroke Apical Lung Examination Study: A Diagnostic and Prognostic Imaging Biomarker in Suspected Acute Stroke
}

(D). Siddiqui, (D) F. Bala, (DS. Sciacca, (D) A.M. Falzon, (DM. Benger, (D) S.A. Matloob, (DF.N.A.C. Miller, (D) R.J. Simister, (D). Chatterjee, (D)L.K. Sztriha, (D). Davagnanam, and (D)T.C. Booth

\begin{abstract}
BACKGROUND AND PURPOSE: Diagnosis of coronavirus disease 2019 (COVID-19) relies on clinical features and reverse-transcriptase polymerase chain reaction testing, but the sensitivity is limited. Carotid CTA is a routine acute stroke investigation and includes the lung apices. We evaluated CTA as a potential COVID-19 diagnostic imaging biomarker.
\end{abstract}

MATERIALS AND METHODS: This was a multicenter, retrospective study $(n=225)$ including CTAs of patients with suspected acute stroke from 3 hyperacute stroke units (March-April 2020). We evaluated the reliability and accuracy of candidate diagnostic imaging biomarkers. Demographics, clinical features, and risk factors for COVID-19 and stroke were analyzed using univariate and multivariate statistics.

RESULTS: Apical ground-glass opacification was present in 22.2\% (50/225) of patients. Ground-glass opacification had high interrater reliability (Fleiss $\kappa=0.81 ; 95 \% \mathrm{Cl}, 0.68-0.95$ ) and, compared with reverse-transcriptase polymerase chain reaction, had good diagnostic performance (sensitivity, 75\% [95\% Cl, 56-87]; specificity, 81\% [95\% Cl, 71-88]; OR = 11.65 [95\% Cl, 4.14-32.78]; P<.001) on multivariate analysis. In contrast, all other contemporaneous demographic, clinical, and imaging features available at CTA were not diagnostic for COVID-19. The presence of apical ground-glass opacification was an independent predictor of increased 30-day mortality $(18.0 \%$ versus $5.7 \%, P=.017$; hazard ratio $=3.51 ; 95 \% \mathrm{Cl}, 1.42-8.66 ; P=.006)$.

CONCLUSIONS: We identified a simple, reliable, and accurate COVID-19 diagnostic and prognostic imaging biomarker obtained from CTA lung apices: the presence or absence of ground-glass opacification. Our findings have important implications in the management of patients presenting with suspected stroke through early identification of COVID-19 and the subsequent limitation of disease transmission.

ABBREVIATIONS: BSTI = British Society of Thoracic Imaging; COVID-19 = coronavirus disease 2019; GGO = ground-glass opacification; IRR = interrater reliability; RT-PCR = reverse-transcriptase polymerase chain reaction; SARS-CoV-2 = Severe Acute Respiratory Syndrome coronavirus 2

$\mathbf{T}$ he Severe Acute Respiratory Syndrome coronavirus 2 (SARSCoV-2) was given pandemic status by the World Health Organization in March 2020. ${ }^{1,2}$ When symptomatic, coronavirus disease 2019 (COVID-19) typically causes mild, self-limiting

Received July 1, 2020; accepted after revision August 17.

From the Departments of Neuroradiology (J.S., F.B., T.C.B.), Neurology (M.B., L.K.S.), and Radiology (F.N.A.C.M.), King's College Hospital, National Health Service Foundation Trust, London, UK; Lysholm Department of Neuroradiology (S.S., A.M.F., I.D.) and Department of Neurosurgery (S.A.M.), National Hospital for Neurology and Neurosurgery, University College London Hospitals, National Health Service Foundation Trust, London, UK; Comprehensive Stroke Service (R.J.S., I.C.), University College London Hospitals, National Health Service Foundation Trust, London, UK; Stroke Research Centre (R.J.S.), and Brain Repair and Rehabilitation Unit (I.D.), University College London Queen Square Institute of Neurology, London, UK; and School of Biomedical Engineering and Imaging Sciences (T.C.B.), King's College London, Rayne Institute, St. Thomas' Hospital, London, UK.

J. Siddiqui and F. Bala are joint first authors and have contributed equally to this work. This work was supported by the Wellcome/Engineering and Physical Sciences Research Council Center for Medical Engineering (WT 203148/Z/16/Z). respiratory features. However, a severe lower respiratory and multisystem disease may occur, necessitating hospitalization. ${ }^{3}$ Approximately $6.0 \%$ of patients with COVID-19 die, and $12 \%$ require intensive care support. ${ }^{4-7}$

Symptoms alone are insufficient for a diagnosis due to a high prevalence of asymptomatic carriers and a variable presymptomatic incubation period (2-14 days). ${ }^{8,9}$ The diagnostic reference standard is the reverse-transcriptase polymerase chain reaction

Please address correspondence to Thomas C. Booth, PhD, School of Biomedical Engineering and Imaging Sciences, King's College London, Rayne Institute, 4th Floor, Lambeth Wing, St. Thomas' Hospital, London SEl 7EH, UK; e-mail: thomas.booth@kcl.ac.uk; @ThomasCBooth

\footnotetext{
- Indicates open access to non-subscribers at www.ajnr.org

Indicates article with supplemental on-line appendix and table.

Indicates article with supplemental on-line photo

http://dx.doi.org/10.3174/ajnr.A6832
} 
(RT-PCR) test using nasopharyngeal swabs or bronchial secretions, but it is constrained by a sensitivity of $60 \%-73 \%{ }^{1-12}$ and a timescale of hours to yield results, with no point-of-care test widely available. A dedicated chest CT is likely to be more sensitive than RT-PCR for COVID-19, which, according to 1 study of 1014 patients, has a sensitivity of $88 \%$ compared with $59 \% .{ }^{11}$ However, patients with suspected stroke are not always suspected of having COVID-19. Furthermore, in many health care systems, including in the United States and the United Kingdom, the routine use of chest CT for COVID-19 diagnosis is not feasible or recommended in patients suspected of having COVID-19. ${ }^{13,14}$

The classic COVID-19 appearance on chest CT is groundglass opacification (GGO), with a predilection for the lower lobes and posterior segments. ${ }^{15-18}$ While lung apices are included on carotid CTA during acute-stroke investigations, they may be overlooked when vascular causes of stroke are the focus. ${ }^{19}$ Abdominal CT has shown lower lobe pulmonary abnormalities consistent with COVID-19 in those with a low clinical suspicion for infection. ${ }^{20,21}$ However, there is no evidence determining the prevalence of such findings in the lung apices, nor the relationship between the extent of lung included on CTA and diagnostic accuracy, nor whether COVID-19 diagnostic imaging biomarkers can be obtained from CTA. This is particularly pertinent because COVID-19 appears to be associated with TIAs and strokes. ${ }^{22-26}$

This multicenter, observational study aimed to derive diagnostic imaging biomarkers using CTA to facilitate early identification of patients with COVID-19.

\section{MATERIALS AND METHODS \\ Study Design}

The National Health Research Authority and Research Ethics Committee approved this study. The authors declare no conflicts of interest. There is no overlap in subjects from prior publications.

We identified, retrospectively, adult patients (18 years of age or older) undergoing a CT of the head and CTA for acute stroke investigations at 3 hyperacute stroke units, King's College Hospital, Princess Royal University Hospital, and University College London Hospitals from March 25, 2020, to April 24, 2020 (corresponding to the time and location of the United Kingdom COVID-19 epicenter). We excluded CTAs that were nondiagnostic or performed for nonacute reasons. For reference, a control group of the same number of patients undergoing acute-stroke investigations during the same period in 2019 was also reviewed.

\section{Imaging}

The acute-stroke imaging protocol consisted of noncontrast CT of the head followed by a craniocervical arterial phase acquisition after intravenous injection of $50 \mathrm{~mL}$ of iohexol, $647 \mathrm{mg} / \mathrm{mL}(5 \mathrm{~mL} / \mathrm{s})$ (Omnipaque 300; GE Healthcare); $1 \mathrm{~mL} / \mathrm{kg}$ of iohexol, $647 \mathrm{mg} / \mathrm{mL}$ (4 mL/s) (Omnipaque 300; GE Healthcare); or $50 \mathrm{~mL}$ of iohexol, $755 \mathrm{mg} / \mathrm{mL}$ (4 mL/s) (Omnipaque 350; GE Healthcare). The scans were performed on multidetector CT scanners (Optima 660, 64section; Discovery 750, 128-section [both GE Healthcare]; Aquilion Prime 320 slice (Toshiba); Definition AS, 128-section [Siemens]).

The main scanning parameters were as follows for CT of the head/CTA studies respectively: tube voltage $=140 \mathrm{kV}($ peak $) / 100$ $\mathrm{kVp}$; automatic tube current modulation $=100-515 / 5-480 \mathrm{mAs}$; pitch $=0.55 / 0.8 \mathrm{~mm}$; matrix $=512 \times 512$ (both); section thickness $=5 / 0.625 \mathrm{~mm}$; FOV $=230 \times 230 \mathrm{~mm}$ (both). All images were then reconstructed with a section thickness of $0.625 \mathrm{~mm}$, with the same increments.

\section{Data Collection}

Blinded to radiologic findings, we obtained demographic and clinical data from electronic medical records (Sunrise; Epic Electronic Health Records). Data included respiratory and stroke clinical features on admission, risk factors for stroke and COVID-19 transmission, and clinical outcome. The details of RT-PCR testing were also recorded (Altona Diagnostics, Hologic, and Abbott Laboratories).

All imaging examinations were independently evaluated by fellowship-trained neuroradiologists, (J.S., F.B., S.S., A.M.F., with 7, 6, 6 , and 3 years' experience, respectively), blinded to the clinical data and the RT-PCR results. Readers analyzed the images on the PACS using source images, maximum intensity projections, and multiplanar reformatting. The coronal depth of the lung apices imaged was measured for both lungs. CTA studies were reviewed in the axial plane using lung window and vascular window settings (window width/level, 1500/-500 Hounsfield units [HU] and 600/ $150 \mathrm{HU}$, respectively).

To find diagnostic imaging biomarkers for COVID-19 that would be easy to apply by radiologists who are not specialized in chest radiology, we evaluated the presence of GGO and any other pulmonary finding within the lung apices on CTA. Cases in which GGO was unequivocally due to dependency, breathing artifacts, or expiratory change were excluded. GGO was also subclassified as focal or diffuse, central or peripheral, and unilateral or bilateral.

We also used categories based on the British Society of Thoracic Imaging (BSTI) COVID-19 Guidance for Reporting Radiology (a nonvalidated guideline implemented at the start of COVID-19; On-line Table 1) (https://www.bsti.org.uk/media/resources/files/ BSTI_COVID-19_Radiology_Guidance_version_2_16.03.20. pdf). All readers, along with a thoracic radiologist with 18 years' experience as a reference standard reader, gave a 3-point likelihood score to rate the lesions (COVID-19-typical lesions, indeterminate lesions, and non-COVID-19 lesions).

\section{Statistics}

Descriptive statistics were used to summarize data.

We determined the agreement between measures from multiple independent readers using the Fleiss $\kappa$ coefficient with standard interpretation guidelines. ${ }^{27,28} \mathrm{We}$ selected features for which there was "substantial" (0.61-0.80) or "almost perfect" agreement (0.81-1.0) for further accuracy evaluation using confusion matrix outcomes.

Comparative statistics were used to analyze differences in 2020 and 2019 baseline data. Normality of distributions was assessed using the Shapiro-Wilk test. For univariate analyses, we used $\chi^{2}$ tests for categoric variables (or Fisher exact tests when cell frequency was $<5$ ) and the Student $t$ test for continuous data (or the Mann-Whitney $U$ test for non-normal distributions). With regard to the number of events in our study, we were limited by the number of confounders we could include in the multivariate logistic regression, so we followed the recommendation that a minimum 

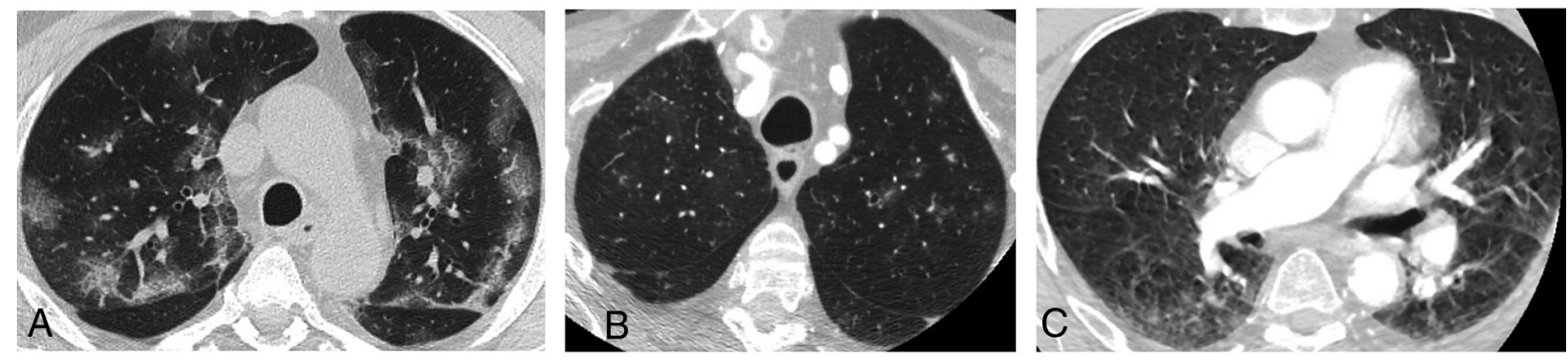

FIG 1. A, There are multiple, bilateral, focal, peripheral-predominant areas of ground-glass opacification, commonly seen in patients with COVID-19 pulmonary infection. $B$, There are multiple, focal 5- to 6-mm nodules with surrounding ground-glass change in the left upper lobe. This is an indeterminate COVID-19 appearance and would be more suggestive of atypical or fungal infection. $C$, There is bilateral ground-glass opacification posteriorly, more on the right, consistent with dependent change. This is a characteristic appearance, not associated with COVID19 , and classically disappears in the prone position. There is also a general hazy appearance not seen in $A$ and $B$, which does not represent abnormal lung and is artifactual due to movement.

of 10 events per variable be incorporated to maintain model validity. ${ }^{29,30} P<.05$ was considered statistically significant.

We also evaluated the relationship between the presence of apical GGO and a craniocaudal measurement of the lung included on the coronal CT scan, by calculating the point biserial correlation coefficient. GGO was tested as a predictor of survival using a multivariate Cox proportional hazards regression model. Statistical analysis was performed using SPSS Statistics (Version 26.0; IBM).

\section{RESULTS}

\section{Baseline Characteristics}

Two hundred twenty-five patients were identified during the 2020 COVID-19 period (On-line Figure $A$ ). The patients' baseline characteristics in 2019 and 2020 were described and compared (On-line Table 2). The mean hospital stay of survivors was shorter in 2020 (9.7 versus 4.4 days; $P=.04$ ). There was no overall difference in clinical and imaging stroke-severity scores, thrombolysis, thrombectomy, or mortality rates.

\section{Imaging Characteristics}

We observed apical GGO in $22.2 \%$ (50/225) of patients in 2020. In 2020, when using the descriptive COVID-19 CT grading system, $28.0 \%$ (14/50) of those with GGO were rated as COVID-19-typical; $24.0 \%$ (12/50), indeterminate; and 48.0\% (24/50), non-COVID-19; all features were more common than in $2019(P<.001)$ (Fig 1).

To ensure that these potential diagnostic imaging biomarkers were reliable, we determined the interobserver agreement of these CTA findings in the setting of a neuroradiology department. The interrater reliability (IRR) was almost perfect (Fleiss $\kappa=0.81 ; 95 \%$ CI, 0.68-0.95) for rating of the presence or absence of apical GGO (On-line Table 3). By means of a descriptive COVID-19 CT grading system, the IRR among 4 neuroradiologist raters was substantial (Fleiss $\kappa=0.74 ; 95 \%$ CI, 0.64-0.84). The IRR was lower but remained substantial when adding a reference standard rating (Fleiss $\kappa=0.65$; 95\% CI, 0.60-0.71). When we reduced the 3 point scale to a 2-point scale (COVID-19-typical/indeterminate versus non-COVID-19/normal), the IRR improved to almost perfect among the neuroradiologists (Fleiss $\kappa=0.88$; 95\% CI, $0.75-$ 1.0 ), and it also improved when adding a reference standard rating (Fleiss $\kappa=0.79$; 95\% CI, 0.71-0.86). Nonetheless, the IRR was highest among neuroradiologist raters for the well-defined tasks of looking at apical GGO subfeatures: focal versus nonfocal (diffuse/ normal), bilateral versus nonbilateral (unilateral/normal), and peripheral versus nonperipheral (central/normal), which gave Fleiss $\kappa$ scores of 0.90 (95\% CI, 0.80-1.00), 0.98 (95\% CI, 0.87-1.0), and 0.87 (95\% CI, 0.78-0.98) respectively.

\section{RT-PCR Cohort Characteristics}

Forty-seven percent (106/225) of patients underwent RT-PCR testing, and $26.4 \%$ (28/106) had positive findings (On-line Table 4). A test was performed at or immediately before CTA in $44.3 \%$ (47/ 106) of patients; findings in $23.4 \%$ (11/47) were positive, but the result was available at the time of CTA in only $14.9 \%$ (7/47). The patients with positive findings on RT-PCR had a higher mortality rate $(28.6 \%[8 / 28]$ versus $7.7 \%[6 / 78], P=.009)$ and received intravenous thrombolysis more often $(32.1 \%$ [9/28] versus $12.8 \%$ [10/ 78 ], $P=.02$ ). There was no difference in any demographic or clinical characteristics, with the exception of oxygen saturation, which was lower in patients with a positive RT-PCR result $(P=.03)$. In contrast, all imaging biomarkers were seen more commonly in patients with a positive RT-PCR result $(P<.001)$. We determined the diagnostic accuracy of these imaging biomarkers for SARSCoV-2 using RT-PCR as the reference standard, having shown their reliability (interrater analyses above).

We measured the sensitivity, specificity, and positive predictive and negative predictive values. The presence of GGO was the most sensitive biomarker, and the descriptive COVID-19 CT grading system using a 2-point scale was the most specific (Table 1). Because the presence of GGO was the most sensitive diagnostic imaging biomarker and the simplest for a clinician to use with high interrater reliability, we incorporated this variable into a multivariate analysis (Table 2), comparing it with other clinical and imaging features immediately available on hospital admission. We included all features in which univariate analysis (On-line Table 4) showed group comparison differences of $P \leq .05$. Only the presence of GGO showed an increased likelihood of a positive RT-PCR result $(\mathrm{OR}=11.65 ; 95 \% \mathrm{CI}, 4.14-32.78 ; P<.001)$.

In summary, radiologists who were not specialized in chest radiology evaluated the presence or absence of apical GGO. This measurement had a very high IRR (Fleiss $\kappa=0.81$; 95\% CI, $0.68-$ 0.95 ) and, when compared with the RT-PCR result, had good COVID-19 diagnostic performance with a sensitivity of $75 \%$ 


\begin{tabular}{|c|c|c|c|c|}
\hline & Sensitivity (95\% Cl) & Specificity $(95 \% \mathrm{CI})$ & PPV $(95 \% \mathrm{Cl})$ & NPV $(95 \% \mathrm{CI})$ \\
\hline Presence of GGO & $75 \%(56-87)$ & $81 \%(71-88)$ & $58 \%(41-74)$ & $90 \%(79-95)$ \\
\hline Focal GGO & $46 \%(29-64)$ & $90 \%(81-95)$ & $62 \%(39-81)$ & $82 \%(72-89)$ \\
\hline Bilateral GGO & $57 \%(37-75)$ & $82 \%(72-89)$ & $53 \%(35-71)$ & $84 \%(73-91)$ \\
\hline Peripheral GGO & $68 \%(49-82)$ & $88 \%(79-94)$ & $68 \%(48-83)$ & $88 \%(79-94)$ \\
\hline COVID-19-typical/in-determinate ${ }^{a}$ & $64 \%(46-79)$ & $92 \%(84-94)$ & $77 \%(50-92)$ & $88 \%(74-95)$ \\
\hline
\end{tabular}

Note:-PPV indicates positive predictive value; NPV, negative predictive value.

${ }^{a}$ COVID-19-typical alone gave a low sensitivity (36\%) and was excluded from further analysis. COVID-19 CT imaging definitions are listed in On-line Table 1.

Table 2: Multivariate analysis using clinical and imaging features to determine the likelihood of SARS-CoV-2 $(n=106)^{\mathrm{a}}$

\begin{tabular}{lccc}
\hline & OR & OR $(95 \% \mathrm{Cl})$ & $P$ Value \\
\hline Infarct & 0.47 & $0.13-1.72$ & .25 \\
Oxygen saturation & 1.00 & $0.97-1.02$ & .78 \\
Presence of GGO & 11.65 & $4.14-32.78$ & $<.001^{\mathrm{b}}$ \\
\hline
\end{tabular}

a With regard to total number of patients with confirmed SARS-CoV-2 (28/106), only the 3 most discriminant characteristics from the univariate analysis $(P \leq .05)$ were included. If all 6 variables $P \leq .10$ or all 10 variables $P \leq .20$ were included in the model, the only significant predictor of positive PCR result was the presence of GGO $(P<.001)$, but these findings are at an increased risk of bias in the OR estimation.

${ }^{\mathrm{b}} \mathrm{P}$ value $<.05$

(95\% CI, 56-87) and a specificity of $81 \%(95 \% \mathrm{CI}, 71-88)$ and OR $=11.65$ (95\% CI, 4.14-32.78; $P<.001)$ on multivariate analysis. While the presence of GGO alone is the simplest feature to understand and measure, we determined that GGO subfeatures (peripheral, focal, or bilateral) or a descriptive grading system were more specific but less sensitive. The grading system gave the highest specificity of $92 \%$ (95\% CI, 84-94).

\section{Apical GGO Cohort Characteristics}

We also examined whether apical GGO (On-line Figure $B$ ), regardless of RT-PCR results, revealed useful information (On-line Table 5). Patients with GGO versus patients without GGO, stroke parameters differed in patients with GGO (50/225) with a higher rate of carotid occlusion on CTA (16.0\% versus $3.4 \%, P=.004)$ and a greater clinical deficit (mean NIHSS score, 6.2 versus $9.4 ; P=.02$ ). In terms of clinical features associated with pneumonia, patients with GGO more often had a cough $(P=.002)$, fever $(P=.004)$, a higher respiratory rate $(P=.005)$, and lower oxygen saturation $(P$ $=.001)$. Patients with GGO had a higher mortality rate $18.0 \%$ (9/ $50)$ versus $5.7 \%(10 / 175)(P=.02)$, and survivors stayed longer in the hospital (3.8 versus 6.6 days, $P=.004$ ). We performed a multivariate analysis (On-line Table 6) incorporating all demographic, clinical, and imaging features immediately available on hospital admission when univariate analysis (On-line Table 5) had shown a group comparison difference of $P<.01$. Carotid occlusion, subjective fever, and lower oxygen saturation showed an increased likelihood of GGO. Carotid occlusion and oxygen saturation were the most predictive features $(\mathrm{OR}=6.82 ; 95 \% \mathrm{CI}, 1.97-23.53 ; P=.002$; $\mathrm{OR}=0.81 ; 95 \%$ CI, 0.69-0.95; $P=.009$, respectively).

Biomarker group differences were not due to a difference in the craniocaudal extent of the lung included on CTA (Table 1 and Online Table 5). Furthermore, during the limited range assessed (mean, $8.06 \pm 2.5 \mathrm{~cm}$ ), there was no correlation between this coronal measurement and the presence of GGO (point biserial correlation coefficient $=0.053$ ); thus, we were unable to show an incentive to increase the extent of apical lung included during CTA.

The presence of GGO was an independent predictor of increased 30 -day mortality ( $18.0 \%$ versus $5.7 \%, P=.02$; hazard ratio $=3.51[95 \% \mathrm{CI}, 1.42-8.66], P=.006$; Fig 2 and On-line Tables 5-7).

\section{DISCUSSION}

Early identification of patients with COVID-19 is essential for treatment and viral control. Therefore, the search for alternative diagnostic biomarkers for COVID-19 is mandated in the context of asymptomatic and presymptomatic infection and the variable sensitivity of SARS-CoV-2 RT-PCR testing. ${ }^{8-12}$ We have assessed candidate biomarkers in those patients who present with suspected acute stroke and undergo immediate CTA. We have shown that an imaging feature, the presence or absence of apical GGO, which is simple to assess, is a reliable and accurate COVID-19 diagnostic biomarker. In contrast, all other contemporaneous demographic, clinical, and imaging features available at the time of CTA were not helpful in the early identification of COVID-19. Furthermore, we have shown that the same biomarker is a prognostic biomarker predictive of 30day mortality. All our findings have not been reported previously.

RT-PCR testing was performed at or before CTA in 44\% (47/ $106)$ of patients, and $23 \%$ (11/47) of tests had positive findings. SARS-CoV-2 RT-PCR testing has limitations as a reference standard due to the diagnostic performance of the assay and external factors related to sampling performance, location, and timing. ${ }^{11}$ Even with many health care systems now adopting routine RT-PCR screening on admission, there may be a relatively long processing time. ${ }^{11}$ Indeed, in our cohort, results were available in only $15 \%$ (7/47) of cases at the time of CTA, thus emphasizing the utility of any reliable alternative opportunisticallyderived diagnostic COVID-19 biomarkers.

Given that the presence of apical GGO was simple to assess and a reliable and accurate diagnostic biomarker for COVID-19, we examined whether patients with apical GGO, regardless of whether a RT-PCR test had been performed, also revealed useful information on admission. GGO was an independent predictor of increased 30 -day mortality $(18.0 \%$ versus $5.7 \% . P=.017$; hazard ratio $=3.51$; $95 \% \mathrm{CI}, 1.42-8.66 ; P=.006)$. A contributory mechanism to this COVID-19-related excess mortality might be thromboembolic because increased carotid occlusion was associated with GGO (16.0\% versus 3.4\%, $P=.004$; OR $=6.82$; 95\% CI, 1.97-23.53; $P=$ .002 ) and was likely an independent predictor of death. This is concordant with reports that COVID-19 is prothrombotic with a putative increase in patients presenting with TIAs and strokes. ${ }^{22-26}$ An 


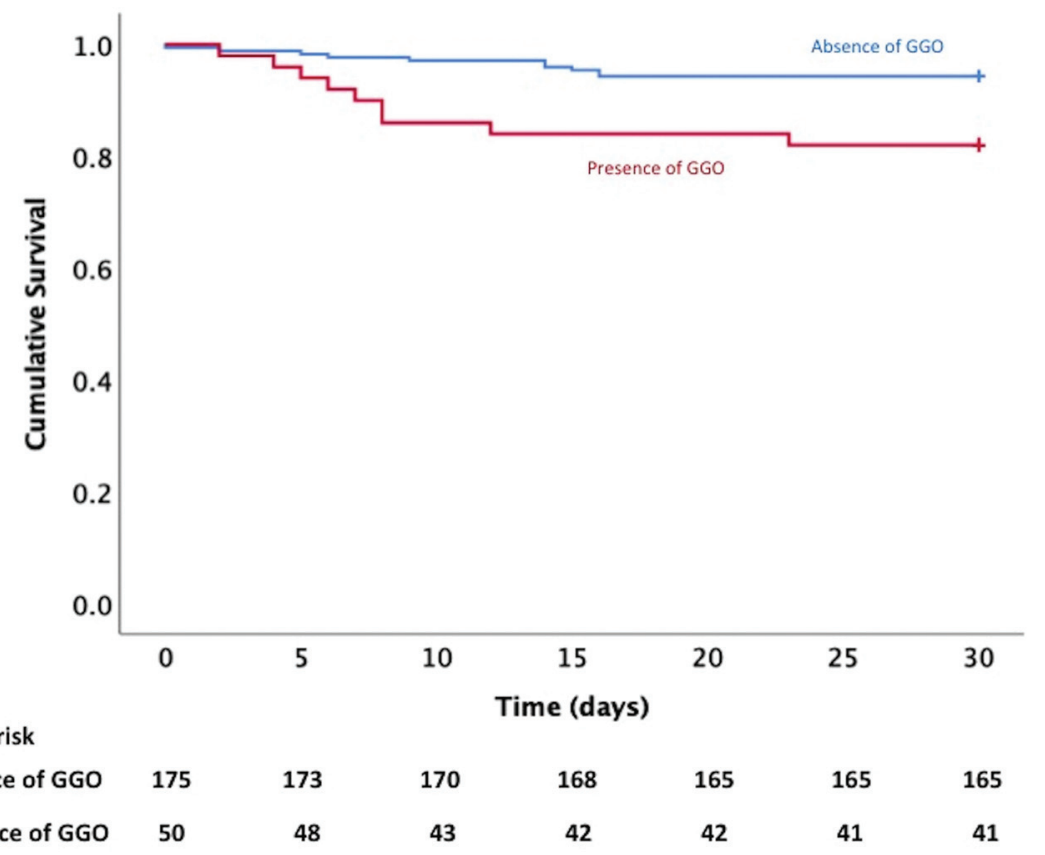

FIG 2. Kaplan-Meier analysis. Association between GGO and mortality was evaluated using Kaplan-Meier survival analysis; $82.0 \%$ of patients in the group with GGO were alive at 30 days compared with $94.3 \%$ in the group without $\mathrm{GGO}(P=.005)$.

implication is that GGO may not be an entirely incidental finding in this cohort of patients with suspected acute stroke. We also noted that unlike patients grouped by RT-PCR results, survivors stayed in the hospital longer $(P=.004)$, plausibly because of the morbidity associated with pneumonitis (or other causes because this is a multisystem disease).

The study most similar to ours analyzed 118 CTA studies performed as stroke investigations and found that 28\% (33/118) had lung findings typical for COVID-19 and 93.9\% (31/33) had a positive SARS-CoV-2 RT-PCR infection. ${ }^{31}$ Another small retrospective study showed apical lung abnormalities on craniocervical CTA in 10/17 (58/8\%) patients with COVID-19 pneumonia whose diagnosis was unknown at the time of CT scanning. ${ }^{32}$ Again, these studies emphasize the importance of careful scrutiny of the lung apices. Our study sampled a greater number of patients than both of these, demonstrating the frequency of ground-glass opacification similar to that in the first study; however, it also included statistics on interrater reliability, diagnostic performance of the biomarker, and survival data, which were not included in either of these studies.

A further study analyzed 119 nonchest CT scans, including abdominal $(n=101)$ and cervical spine $(n=18)$ imaging. ${ }^{21}$ Fifty-two percent (62/119) of patients were suspected of having COVID-19 and underwent RT-PCR testing before the CT reading; 48\% (57/119) were not suspected of having COVID-19. The presence of pneumonia was seen in 64\% (76/119), leading to a diagnosis of COVID-19 in $37 \%$ (44/119). The prevalence of pneumonia was higher (64\%) than the presence of apical GGO in our cohort (22\%), plausibly due to a broader definition of COVID-19-typical abnormality and 102/119 patients (84\%) undergoing abdominal imaging, including the lung bases. The lung bases likely yield more COVID-19-typical abnormalities than the apices, given the predilection for lower lobe and posterior segment involvement. ${ }^{15-18}$ The diagnostic reliability and accuracy metrics including IRR, sensitivity, and specificity were not reported.

The implications of our findings plausibly include earlier selection of the appropriate level of personal protective equipment and attendant staff numbers, triage to appropriate inpatient ward settings, self-isolation, and contact tracing. ${ }^{33}$ Guidance on selection of personal protective equipment often changes on the basis of available evidence, and risk stratification is the key to this in a number of health care settings. ${ }^{34,35}$ Biomarkers, such as a scan positive for GGO, should heighten awareness of a potential positive case, possibly changing staff personal protective equipment requirements (eg, from a fluid-repellant surgical mask to an FFP2 or N95 mask) and also directing a patient to a side room instead of an open ward, pending RT-PCR results. This patient group may additionally find it difficult to wear a mask, hence increasing the importance of staff protection. Our data therefore have important safety implications for daily clinical practice as well as prognostic information, given the increased mortality in those with COVID-19 shown in our cohort.

Our study has a number of strengths. It is multicenter and is the only study robustly assessing the apices for GGO. Its main limitation is its retrospective nature; however, no prospective studies exist in the literature relating to COVID-19 imaging. The use of the nonvalidated BSTI COVID-19 guidelines in some parts of the study may also be a limitation; however, no validated pulmonary scoring system yet exists for COVID-19 nor does this issue interfere with our conclusions.

\section{CONCLUSIONS}

This study demonstrates that the presence of apical GGO on carotid CTA in patients presenting with suspected acute stroke is a simple, reliable, and accurate diagnostic and prognostic COVID-19 biomarker. This can now be tested prospectively for further validation. These findings mandate vigilance in apical assessment by all radiologists and clinicians involved in acute stroke care, particularly relevant given the sensitivity of currently available SARS-CoV-2 RTPCR testing.

\section{ACKNOWLEDGMENTS}

The authors acknowledge the South London Specialist Virology Centre, Viapath, Infections Sciences, in this study.

\section{REFERENCES}

1. World Health Organization. Naming the coronavirus disease (COVID-19) and the virus that causes it. https://www.who.int/ 
emergencies/diseases/novel-coronavirus-2019/technical-guidance/ naming-the-coronavirus-disease-(covid-2019)-and-the-virus-thatcauses-it. Accessed June 20, 2020

2. World Health Organization. WHO Director-General's opening remarks at the media briefing on COVID-19 - 11 March 2020. WHO Director-General's opening remarks at the media briefing on COVID-19 - 11 March 2020. Accessed June 20, 2020

3. Huang C, Wang Y, Li X, et al. Clinical features of patients infected with 2019 novel coronavirus in Wuhan, China. Lancet 2020;395:497506 CrossRef Medline

4. Grasselli G, Pesenti A, Cecconi M. Critical care utilization for the COVID-19 outbreak in Lombardy, Italy: early experience and forecast during an emergency response. JAMA 2020 Mar 13. [Epub ahead of print] CrossRef

5. Remuzzi A, Remuzzi G. COVID-19 and Italy: what next? Lancet 2020;395:1225-28 CrossRef Medline

6. Phua J, Weng L, Ling L, et al; Asian Critical Care Clinical Trials Group. Intensive care management of coronavirus disease 2019 (COVID19): challenges and recommendations. Lancet Respir Med 2020;8:50617 CrossRef Medline

7. Worldometer. COVID-19 Coronavirus Pandemic. https://www. worldometers.info/coronavirus/. Accessed 20th June, 2020

8. Day M. Covid-19: four fifths of cases are asymptomatic, China figures indicate. $\mathrm{Br} \mathrm{Med} \mathrm{J} \mathrm{2020;369:m1375} \mathrm{CrossRef} \mathrm{Medline}$

9. Lauer SA, Grantz KH, Bi Q, et al. The incubation period of coronavirus disease 2019 (COVID-19) from publicly reported confirmed cases: estimation and application. Ann Intern Med 2020;172:577-82 CrossRef Medline

10. Yang Y, Yang M, Shen C, et al. Evaluating the accuracy of different respiratory specimens in the laboratory diagnosis and monitoring the viral shedding of 2019-nCoV infections. medRXiv. http://medrxiv.org/ lookup/doi/10.1101/2020.02.11.20021493. Accessed June 20, 2020

11. Ai T, Yang Z, Hou H, et al. Correlation of chest CT and RT-PCR testing in coronavirus disease 2019 (COVID-19) in China: a report of 1014 cases. Radiology 2020;296:E32-40 CrossRef Medline

12. Fang Y, Zhang H, Xie J, et al. Sensitivity of chest CT for COVID19: comparison to RT-PCR. Radiology 2020;296:E115-17 CrossRef Medline

13. American College of Radiology. ACR Recommendations for the Use of Chest Radiography and Computed Tomography (CT) for Suspected COVID-19 Infection. https:/www.acr.org/Advocacy-and-Economics/ ACR-Position-Statements/Recommendations-for-Chest-Radiographyand-CT-for-Suspected-COVID19-Infection. Accessed June 20, 2020

14. The Royal College of Radiologists. The role of CT chest during the COVID-19 crisis. https://www.rcr.ac.uk/college/coronavirus-covid-19what-rcr-doing/clinical-information/role-ct-chest-during-covid19. Accessed June 20, 2020

15. Wong HY, Lam HYS Fong AH, et al. Frequency and distribution of chest radiographic findings in COVID-19 positive patients. Radiology 2020;296:E72-78 CrossRef Medline

16. Chung M, Bernheim A, Mei X, et al. CT imaging features of 2019 novel coronavirus (2019-nCoV). Radiology 2020;295:202-07 CrossRef Medline

17. Bernheim A, Mei X, Huang M, et al. Chest CT findings in coronavirus disease-19 (COVID-19): relationship to duration of infection. Radiology 2020;295:200463 CrossRef Medline
18. Caruso D, Zerunian M, Polici M, et al. Chest CT features of COVID19 in Rome, Italy. Radiology 2020;296:E79-85 CrossRef Medline

19. Kanesa-Thasan R, Cox M, Patel M, et al. Actionable vascular and other incidental findings on CTA in patients undergoing acute stroke intervention. Neuroradiol J 2018;31:572-77 CrossRef Medline

20. Vu D, Ruggiero M, Choi WS, et al. Three unsuspected CT diagnoses of COVID-19. Emerg Radiol 2020;27:229-32 CrossRef Medline

21. Hossain R, Lazarus MS, Roudenko A, et al. CT scans obtained for nonpulmonary indications: associated respiratory findings of COVID19. Radiology 2020;296:E173-79 CrossRef Medline

22. Klok FA, Kruip MJ, van der Meer NJ, et al. Incidence of thrombotic complications in critically ill ICU patients with COVID-19. Thromb Res 2020;191:145-47 CrossRef Medline

23. Mao L, Jin H, Wang M, et al. Neurologic manifestations of hospitalized patients with coronavirus disease 2019 in Wuhan, China. JAMA Neurol 2020;77:683-89 CrossRef Medline

24. Helms J, Tacquard C, Severac F, et al; CRICS TRIGGERSEP Group (Clinical Research in Intensive Care and Sepsis Trial Group for Global Evaluation and Research in Sepsis). High risk of thrombosis in patients with severe SARS-CoV-2 infection: a multicenter prospective cohort study. Intensive Care Med 2020;46:1089-98 CrossRef Medline

25. Connors JM, Levy JH. Thromboinflammation and the hypercoagulability of COVID-19. J Thromb Haemost 2020;18:1559-61 CrossRef Medline

26. Oxley TJ, Mocco J, Majidi S, et al. Large-vessel stroke as a presenting feature of covid-19 in the young. N Engl J Med 2020;382:e60 CrossRef Medline

27. Fleiss JL. Measuring nominal scale agreement among many raters. Psychol Bull 1971;76:378-82 CrossRef

28. Landis JR, Koch GG. The measurement of observer agreement for categorical data. Biometrics 1977;33:159-74 Medline

29. Harrell FE Jr, Lee KL, Matchar DB, et al. Regression models for prognostic prediction: advantages, problems, and suggested solutions. Cancer Treat Rep 1985;69:1071-77 Medline

30. Peduzzi P, Concato J, Kemper E, et al. A simulation study of the number of events per variable in logistic regression analysis. J Clin Epidemiol 1996;49:1373-79 CrossRef Medline

31. Kihira S, Schefflein J, Chung M, et al. Incidental COVID-19 related apical lung findings on stroke CTA during the COVID-19 pandemic. J Neurointerv Surg 2020;12:669-72 CrossRef Medline

32. Applewhite $\mathrm{BP}$, Buch $\mathrm{K}$, Yoon $\mathrm{BC}$, et al. Lung apical findings in coronavirus disease (COVID-19) infection on neck and cervical spine CT. Emegr Radiol 2020 Jul 21. [Epub ahead of print] CrossRef Medline

33. Public Health England. COVID-19: Guidance for the remobilisation of services within health and care settings: infection prevention and control recommendations. https://assets.publishing. service.gov.uk/government/uploads/system/uploads/attachment_ data/file/886668/COVID-19_Infection_prevention_and_control_ guidance_complete.pdf. Accessed June 20, 2020

34. Sayburn A. Covid-19: PHE upgrades PPE advice for all patient contacts with risk of infection. $\mathrm{Br}$ Med J 2020;369:m1391 CrossRef Medline

35. Forrester JD, Nassar AK, Maggio PM, et al. Precautions for operating team members during the COVID-19 pandemic. J Am Coll Surg 2020;230:1098-1101 CrossRef Medline 